\title{
UCA1 overexpression is associated with less aggressive subtypes of bladder cancer
}

\author{
LAETITIA LEBRUN $^{1 *}$, DINA MILOWICH ${ }^{1,2^{*}}$, MARIE LE MERCIER $^{1}$, JUSTINE ALLARD $^{1,3}$, \\ YVES-RÉMI VAN EYCKE ${ }^{3,4}$, THIERRY ROUMEGUERE ${ }^{5}$, CHRISTINE DECAESTECKER ${ }^{3,4}$, \\ ISABELLE SALMON ${ }^{1,3,6}$ and SANDRINE RORIVE ${ }^{1,3,6}$
}

\begin{abstract}
${ }^{1}$ Department of Pathology, Erasme University Hospital, Université Libre de Bruxelles (ULB), B-1070 Brussels, Belgium; ${ }^{2}$ Department of Pathology, University Institute of Pathology, Lausanne, CH-1011 Vaud, Switzerland; ${ }^{3}$ DIAPath-Center for Microscopy and Molecular Imaging, ULB, B-6041 Gosselies; ${ }^{4}$ Laboratories of Image, Signal processing and Acoustics, Brussels School of Engineering/Ecole Polytechnic de Brussels, ULB, B-1050 Brussels; ${ }^{5}$ Department of Urology, Erasme University Hospital, ULB, B-1070 Brussels; ${ }^{6}$ Centre Universitaire Inter Regional d'Expertise en Anatomie Pathologique Hospitalière (CurePath),
\end{abstract} B-6040 Charleroi (Jumet), Belgium

Received March 29, 2018; Accepted August 16, 2018

DOI: $10.3892 / o r .2018 .6697$

\begin{abstract}
Non-coding RNAs (ncRNAs) have been shown to serve important roles in carcinogenesis via complex mechanisms, including transcriptional and post-transcriptional regulation, and chromatin interactions. Urothelial carcinoma-associated 1 (UCA1), a long ncRNA, was recently shown to have tumorigenic properties in urothelial bladder cancer (UBC), as demonstrated by enhanced proliferation, migration, invasion and therapy resistance of UBC cell lines in vitro. These in vitro findings suggested that $U C A 1$ is associated with aggressive tumor behavior and could have prognostic implications in UBC. The aims of the present study were to therefore to investigate the statistical associations between UCA1 RNA expression and UBC pathological features, patient prognosis and p53 and Ki-67 expression. Chromogenic in situ hybridization and immunohistochemistry were performed on UBC tissue microarrays to characterize UCA1 RNA, and p53 and $\mathrm{Ki}-67$ expression in $208 \mathrm{UBC}$ cases, including 145 non-muscle-invasive and 63 muscle-invasive cases. UCA 1 was observed in the tumor cells of 166/208 (80\%) UBC cases tested. No expression was noted in normal stromal and endothelium cells. Patients with UBC that overexpressed UCAI $(35 \%)$ had a significantly higher survival rate $(\mathrm{P}=0.006)$
\end{abstract}

Correspondence to: Professor Sandrine Rorive, Department of Pathology, Erasme University Hospital, Université Libre de Bruxelles (ULB), 808 Route de Lennik, B-1070 Brussels, Belgium E-mail: sandrine.rorive@erasme.ulb.ac.be

*Contributed equally

Key words: urothelial carcinoma-associated 1, bladder cancer, prognostic marker, long non-coding RNA, biomarker compared with that in patients with UBC that did not overexpress $U C A 1$. This prognostic factor was independent of tumor morphology, concomitant carcinoma in situ, tumor grade and tumor stage. In addition, the absence of UCA1 overexpression was significantly associated with a high $\mathrm{Ki}-67$ proliferative index $(\mathrm{P}=0.008)$ and a p53 'mutated' immunoprofile (strong nuclear expression or complete absence of staining; $\mathrm{P}=0.003$ ). In conclusion, the present results identified $U C A 1$ as potentially being a novel independent prognostic marker in UBC that was associated with a better patient prognosis and that could serve a pivotal role in bladder cancer carcinogenesis.

\section{Introduction}

Urothelial bladder cancer (UBC) is recorded as the seventh most commonly occurring malignancy in men and the seventeenth most common in women (1). The prevention of risk factors, including smoking and occupational chemicals, has resulted in a decrease of the UBC burden in Western communities; yet, no clinically relevant differences in UBC mortality have been observed over the past 30 years (2).

Standard treatment modalities for UBC have not changed a lot in recent years and encompass intravesical chemotherapy for non-muscle-invasive bladder cancer (NMIBC) and cystectomy with cisplatin-based systemic chemotherapy for MIBC; however, recently, immunotherapy and more precisely, atezolizumab or pembrolizumab, has been approved by the Food and Drug Administration for patients with metastatic disease who progressed during or following cisplatin-based chemotherapy $(1,2)$. Recent insights into the biology of UBC by whole genome, RNA and microRNA sequencing have identified molecular subtypes in MIBC with different putative therapeutic targets and variable sensitivity to currently available chemotherapies $(3,4)$. These studies are part of an ongoing effort to further characterize UBC, using protein and gene expression profiles to identify patients who are at risk for progression or 
recurrence and may benefit from a more aggressive therapeutic approach, as well as to avoid over-treatment of tumors with a relatively indolent clinical course (3). Numerous genes have been implicated in the development and progression of UBC, and their altered expression has been attributed to gene mutations and epigenetic changes. In the latter category, non-coding RNAs (ncRNAs) have been demonstrated to serve important roles in carcinogenesis and cancer metastasis via complex mechanisms, including transcriptional and post-transcriptional regulation, and chromatin interactions (5). The ncRNAs are divided into small regulatory RNAs and long ncRNAs (lncRNAs), composed of $>200$ nucleotides (6). Urothelial carcinoma-associated 1 (UCA1) is an IncRNA that was first isolated by Wang et al (7) in 2006 and was initially proposed as a urinary biomarker for the detection of UBC, although reports about the efficiency and clinical application of UCAI as a diagnostic test have been controversial $(8,9)$. Concurrently, UCAI was shown to have tumorigenic properties, as shown in vitro by enhanced proliferation, invasion, migration and therapy resistance of UBC cell lines $(10,11)$. A subsequent study identified $U C A 1$ as a crucial element in cell cycle regulation by positive indirect action on the phosphoinositide 3-kinase (PI3K)-protein kinase $\mathrm{B}(\mathrm{AKT})$-mechanistic target of rapamycin pathway through the $\mathrm{p} 300$ coactivator cAMP response element-binding protein (CREB) (12). The majority of these results are provided by in vitro cellular assays, and together they suggest that $U C A 1$ is associated with more aggressive tumor behavior and could therefore have prognostic implications in UBC. Indeed, several studies, mainly conducted in Chinese patients with solid tumors (esophageal, gastric, colorectal, prostate, breast, endometrial, ovarian or non-small cell lung carcinoma) reported that a high level of $U C A I$ expression was associated with positive lymph node metastasis, higher clinical stage and poor survival $(13,14)$. To date few and contradictory data are currently available with regard to the expression of $U C A I$ [measured by reverse transcription-quantitative polymerase chain reaction (RT-qPCR)] in series of human UBC and its association with UBC morphology and aggressiveness $(7,15)$. In the present study, chromogenic in situ hybridization (CISH) was applied to analyze the expression of UCAI in human UBC samples, thus integrating expression levels and in vivo morphological context. Additionally, the study aimed to determine the value of $U C A 1$ as a prognostic marker in patients with UBC and its association with other biological markers associated with UBC aggressiveness, including p53, encoded by key tumor suppressor gene TP53, and the proliferation marker Ki-67 (16,17).

\section{Materials and methods}

Ethics statement. The present study was approved by the Ethical Committee of the Erasme University Hospital (Brussels, Belgium; ref., P2015/041). According to Belgian law, no written informed consent was required for archival material in the context of retrospective studies. The ethical committee thus waived the requirement for written informed consent from the participant.

Tissue samples. A total of 11 human UBC tissue microarrays (TMA) and one normal urothelium TMA were manufactured
(MiniCore tissue arrayer; Mitogen Ltd., Harpenden, UK) using available archival formalin-fixed and paraffin-embedded (FFPE, ISO15189 standard) samples from cases of $271 \mathrm{UBC}$ and 40 samples of normal urothelium collected between January 1997 and December 2007 in the Erasme Hospital Biobank (Brussels, Belgium; BE_BERA1; Biobanque Hôpital Erasme-ULB (BERA); BE_NBWB1; Biothèque Wallonie Bruxelles (BWB); BBMRI-ERIC). Cases for which archival material was insufficient or not available were excluded from the analysis. The time period was selected in order to obtain a follow-up of at least 5 years for all patients. All the tumors are from patients who were not previously treated for UBC (primary tumor resection), and whose histopathological diagnoses were reviewed and characterized by an uropathologist. Tumor grades and stages were adjusted to comply with the new 2016 WHO classification (18) and the new 2017 TNM Classification of Malignant Tumours (Union for International Cancer Control) (19) (Table I). For each case, six tissues cores of $600 \mu \mathrm{m}$ in diameter targeting the tumor area (without distinction between the areas of bladder wall) and four tissues cores of $600 \mu \mathrm{m}$ in diameter targeting the normal urothelium were included in the TMAs. Subsequent to validating each TMA for RNA and tissue fixation quality, 208 out of the 271 preselected UBC cases and 20 samples of normal urothelium were included. The available clinical and pathological features of the patients and their tumors are described in Table I. Patient outcomes were characterized in terms of disease-free survival (DFS) and cancer-specific survival (CSS), i.e., periods from the date of the first tumor resection (the date of the diagnosis) until the date of recurrence or mortality (DFS) or the date of mortality due to tumor progression (CSS).

UCA1 CISH. Detection of UCA1 RNA was performed by CISH using the RNAscope ${ }^{\circledR}$ Singleplex Target Probe and the RNAscope 2.0 HD Detection kit (Advanced Cell Diagnostics, Newark, CA, USA). Sections (5- $\mu \mathrm{m}$ thick) of all TMAs were baked at $60^{\circ} \mathrm{C}$ for $1 \mathrm{~h}$ and deparaffinized. Slides were rehydrated and three pre-treat solutions were successively applied according to the manufacturer's recommendations. Following retrieval, UCAl anti-sense probe (RNAscope Singlepex Target Probe), positive (RNAscope Positive Control Probe-PPIB; cat. no. 313901) or negative control probe (RNAscope Negative Control Probe-DapB; cat. no. 310043) were dispensed onto slides at $40^{\circ} \mathrm{C}$ for $2 \mathrm{~h}$. Following hybridization, slides were incubated with six oligonucleotide probes for signal amplification. The first four amplifiers were hybridized at $40^{\circ} \mathrm{C}$ and the last two were incubated at room temperature. Following each hybridization step, slides were washed with a washing buffer two times at room temperature. For chromogenic detection, equal volumes of BROWN-A and BROWN-B DAB substrates from the CISH kit were dropped onto each slide for $10 \mathrm{~min}$ at room temperature. Tissue nuclei were then stained using Gill's hematoxylin (Vector Laboratories, Ltd., Peterborough, UK; cat. no. H-3401) for $2 \mathrm{~min}$ at room temperature. Only cases for which the positive (PPIB) and the negative (bacterial DapB gene, which encodes for the dihydrodipicolinate reductase protein) probes were validated on tissue cores (to ensure the quality of the RNA in the tissue and the absence of false-positive results, respectively) were included in the study. UCAI CISH staining was visualized by using Spot Browser V2e 
Table I. Patient demographics and baseline features $(n=208)$.

\begin{tabular}{|c|c|c|}
\hline Clinical features & NMIBC (n=145) & $\operatorname{MIBC}(n=63)$ \\
\hline Median age (range), years & $69.4(35.4-97)$ & $71.1(33.9-91.3)$ \\
\hline \multicolumn{3}{|l|}{ Sex, n $(\%)$} \\
\hline Male & $118(81.4)$ & $54(85.7)$ \\
\hline Female & $27(18.6)$ & $9(14.3)$ \\
\hline \multicolumn{3}{|l|}{ UBC morphology, n (\%) } \\
\hline Papillary lesions & $140(96.6)$ & $46(73.0)$ \\
\hline Flat lesions & $5(3.4)$ & $17(27.0)$ \\
\hline \multicolumn{3}{|l|}{ UBC variant histology, n (\%) } \\
\hline Present & $11(7.6)$ & $27(42.9)$ \\
\hline Squamous & $3(2.1)$ & $13(20.6)$ \\
\hline Glandular & $2(1.4)$ & $7(11.1)$ \\
\hline Micropapillary & $6(4.1)$ & $8(12.7)$ \\
\hline Sarcomatoid & $1(0.7)$ & $6(9.5)$ \\
\hline Absent & $134(92.4)$ & $36(57.1)$ \\
\hline \multicolumn{3}{|l|}{ Multifocality, n (\%) } \\
\hline Present & $40(27.6)$ & $20(31.7)$ \\
\hline Absent & $95(65.5)$ & $38(60.3)$ \\
\hline Unknown & $10(6.9)$ & $5(8.0)$ \\
\hline \multicolumn{3}{|l|}{ Concomitant CIS, n (\%) } \\
\hline Present & $19(13.1)$ & $30(47.6)$ \\
\hline Absent & $125(86.2)$ & $26(41.3)$ \\
\hline Unknown $^{\mathrm{a}}$ & $1(0.7)$ & $7(11.1)$ \\
\hline \multicolumn{3}{|c|}{ Lymphovascular invasion, n (\%) } \\
\hline Present & $5(3.4)$ & $36(57.1)$ \\
\hline Absent & $109(75.2)$ & $18(28.6)$ \\
\hline Unknown $^{\mathrm{a}}$ & $31(21.4)$ & $9(14.3)$ \\
\hline \multicolumn{3}{|l|}{2016 WHO grading, n (\%) } \\
\hline PUNLMP & $31(21.4)$ & $0(0.0)$ \\
\hline Low grade & $64(44.1)$ & $1(1.6)$ \\
\hline High grade & $50(34.5)$ & $62(98.4)$ \\
\hline \multicolumn{3}{|l|}{ Recurrence, n (\%) } \\
\hline Yes & $55(62.1)$ & $31(49.2)$ \\
\hline No & $55(37.9)$ & $32(50.8)$ \\
\hline \multicolumn{3}{|l|}{ Mortalities, n (\%) } \\
\hline Yes & $4(2.7)$ & $12(19)$ \\
\hline No & $141(97.3)$ & $51(81)$ \\
\hline \multicolumn{3}{|l|}{ Median follow-up (range) } \\
\hline Months & $83.6(0.1-212)$ & $14.1(0.0-189.2)$ \\
\hline Years & $7(0.0-17.7)$ & $1.2(0.0-15.8)$ \\
\hline
\end{tabular}

Numbers (or percentages) of UBC patients included in the study are displayed and describes their clinical and pathological features; cases are

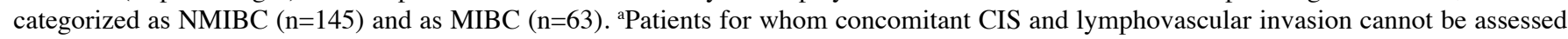
due to coagulation artifacts or small sample size. UBC, urothelial bladder cancer; NMIBC, non-muscle invasive bladder cancer; MIBC, muscle invasive bladder cancer; CIS, carcinoma in situ; WHO, World Health Organization; PUNLMP, papillary urothelial neoplasm of low malignant potential.

(Alphelys, Plaisir, France) and scored by two independent observers using a three-tiered scoring system as follows: 0 , no staining; 1, a few dots observed in a few tumor cells; and $2,>10$ dots homogeneously observed in tumor cells; Fig. 1).
For each UBC case, the mean score of the 6 tissue cores was calculated and the case was categorized as 'UCA1-negative or with low expression' and as 'UCAl-positive or overexpressed' if the mean score was $\leq 1$ and $>1$, respectively. 
Immunohistochemistry (IHC). Sections (5- $\mu \mathrm{m}$ thick) of all TMAs were subjected to standard IHC on a Ventana Discovery XT (Ventana Medical Systems, Inc., Tucson, AZ, USA) using the DABMap detection system according to the manufacturer's protocols. Briefly, the slides were incubated with the rabbit monoclonal anti-Ki-67 antibody for $24 \mathrm{~min}$ at $37^{\circ} \mathrm{C}$ (RTU antibody: $\sim 2 \mu \mathrm{g} / \mathrm{ml}$, clone 30-9; cat. no. 790-4286; Ventana Medical Systems, Inc.) or with the mouse monoclonal anti-p53 antibody for $28 \mathrm{~min}$ at $37^{\circ} \mathrm{C}$ (RTU antibody: $\sim 2.5 \mu \mathrm{g} / \mathrm{ml}$, clone Bp53-11; cat. no. 760-2542; Ventana Medical Systems, Inc.). The slides were washed and incubated with the biotinylated secondary antibody (1:200 dilution; cat. no. BA-2001 for anti-mouse antibody; cat. no. BA-1000 for anti-rabbit antibody; both Vector Laboratories, Ltd.) for $24 \mathrm{~min}$ at $37^{\circ} \mathrm{C}$ and $20 \mathrm{~min}$ at $37^{\circ} \mathrm{C}$, respectively, followed by the addition of complex avidin-horseradish peroxidase. Immunostainings were detected by incubation with diaminobenzidine and hydrogen peroxide. All IHC slides were counterstained with Gill's hematoxylin for $2 \mathrm{~min}$ at room temperature, dehydrated and mounted. For each staining, an external positive control was included as well as a negative control, which entailed replacing the primary antibody with non-immune serum (Dako; Agilent Technologies, Inc., Santa Clara, CA, USA). In addition, anti-vimentin immunostaining (RTU antibody: $\sim 2.5 \mu \mathrm{g} / \mathrm{ml}$, clone V9; cat. no. 790-2917; Ventana Medical Systems, Inc.) for $24 \mathrm{~min}$ at $37^{\circ} \mathrm{C}$ was performed on each TMA for the quality control of tissue fixation. Two independent observers performed the semi-quantitative assessment of p53 IHC expression by using a three-tier score as follows: 0 , no staining; $1, \leq 25 \%$ of cells with weak, heterogeneous cytoplasmic and nuclear staining; and 2, $>25 \%$ of cells with high homogeneous nuclear staining (Fig. 1). For discordant cases, a third pathologist blinded to previous results reclassified the p53 IHC staining. Ki-67 slides were digitalized using a NanoZoomer 2.0 HT (Hamamatsu Photonics K.K., Hamamatsu, Japan) and Ki-67 expression was quantitatively evaluated using Visiomorph DP 5.1 (Visiopharm, Hoersholm, Denmark) in the tumor areas manually selected on the digital slides by a pathologist. The Ki-67 labeling index (Ki-67_LI), corresponding to the ratio between the surface area occupied by the positive nuclei and the total nucleus area, was globally computed for each case (across the 6 cores), as previously detailed $(20,21)$. Based on published data (16), a threshold of $25 \%$ in terms of Ki-67_LI was used for statistical analyses.

Statistical analysis. All statistical analyses were performed using Statistica 12 (StatSoft, Inc., Tulsa, OK, USA). Comparisons between two independent groups of numerical data were performed using the non-parametric Mann-Whitney test. The association between two qualitative variables was assessed using Fisher's exact test or the $\chi^{2}$ test, depending on whether the two variables were binary or not. Univariate survival analyses were performed using a standard Kaplan-Meier analysis and a log-rank test, with the exception of cases of continuous variables, for which univariate Cox regression was used. The analyses were completed using multivariate Cox regression; when analyzing the set of clinical variables, those with univariate results of $\mathrm{P}<0.05$ were selected. The potential contributions of the biological variables were then tested to the final 'clinical' model by adjusting for those for which the univariate results indicated a P-value of $<0.1$. For each statistical analysis, the cases with missing value(s) in the concerned variable(s) were omitted.

\section{Results}

As illustrated in Fig. 1A-C, the CISH methodology detected UCA1 dot expression in the cytoplasm and in the nucleus of urothelial normal and tumor cells. All the normal urothelium cases showed UCA1 dot positivity (data not shown). No UCA1 dot staining was observed in endothelial, stromal or inflammatory cells (Fig. 1A-C). UCA1 dot positivity was detected in 166 out of the 208 UBC included in the present study (80\%). According to the semi-quantitative scoring, UCA1 overexpression (with a positivity mean score of $>1$ ) was observed in 72/208 UBC (35\%), including 55 (76\%) NMIBC [i.e. 32/84 (38\%) pTa and 23/61 (38\%) pT1] and 17 (24\%) MIBC, respectively (Table II). UCA1 overexpression was not statistically associated with tumor morphology (papillary vs. flat lesions), presence of a secondary histological variant (including squamous, sarcomatoid, glandular or micropapillary mixed UBC tumors), tumor multifocality or the presence of concomitant carcinoma in situ (CIS) (Table II). However, UBC overexpressing UCAl was statistically associated with less lymphovascular invasion compared with UBC that failed to overexpress $U C A 1(\mathrm{P}=0.02)$. No statistical association was found between $U C A l$ overexpression and UBC tumor grade (papillary urothelial neoplasm of low malignant potential, low-grade and high-grade tumors) or stage ('pTa, pT1, pT2, pT3, pT4' or 'NMIBC vs. MIBC') (Table II). Regarding p53 expression in UBC, it was noted that the complete absence of p53 expression (i.e., a score of 0; Fig. 1D) and strong and diffuse nuclear p53 staining in $>25 \%$ of UBC cells (i.e., a score of 2; Fig. 1F) followed similar profiles in terms of UBC aggressiveness as opposed to the weak and heterogeneous p53 expression pattern (i.e., a score of 1) (data not shown); these results were similar to those of previous studies regarding p53 expression in serous ovarian and endometrial carcinoma (22-24). The data obtained from UBC associated with a p53 'score 0 ' and a p53 'score 2' were grouped (referred to as the 'p53-mutated immunoprofile'), as opposed to the p53 'score 1' (referred to as the 'p53-wild-type immunoprofile'), for use in future statistical analyses. These analyses showed that, compared with the 'p53-wild-type' immunoprofile, the 'p53-mutated' immunoprofile was observed significantly more in association with flat $\mathrm{UBC}$ morphology $(\mathrm{P}=0.01)$, presence of a secondary histological variant $(\mathrm{P}=0.00001)$, concomitant CIS ( $\mathrm{P}=0.0006)$, lymphovascular invasion $(\mathrm{P}=0.0002)$, UBC tumor high-grade $(\mathrm{P}=0.00001)$ and UBC tumor high-stage ( $\mathrm{P}=0.00003$ ) (Table II). Similarly, a high Ki-67 proliferative index $(>25 \%)$ was observed significantly more in association with more aggressive UBC, i.e., UBC with flat urothelial morphology $(\mathrm{P}=0.04)$, presence of a secondary histological variant $(\mathrm{P}=0.003)$, concomitant $\mathrm{CIS}(\mathrm{P}=0.00003)$, lymphovascular invasion $(\mathrm{P}<0.00001)$, tumor grade $(\mathrm{P}<0.00001)$ and tumor stage $(\mathrm{P}<0.00001$; Table II).

To evaluate the prognostic contribution of UCAl overexpression in patients with UBC, first, the impact of the clinical factors (listed in Table I) on DFS and CSS (Table III) was analyzed. Univariate survival rate analyses revealed that 


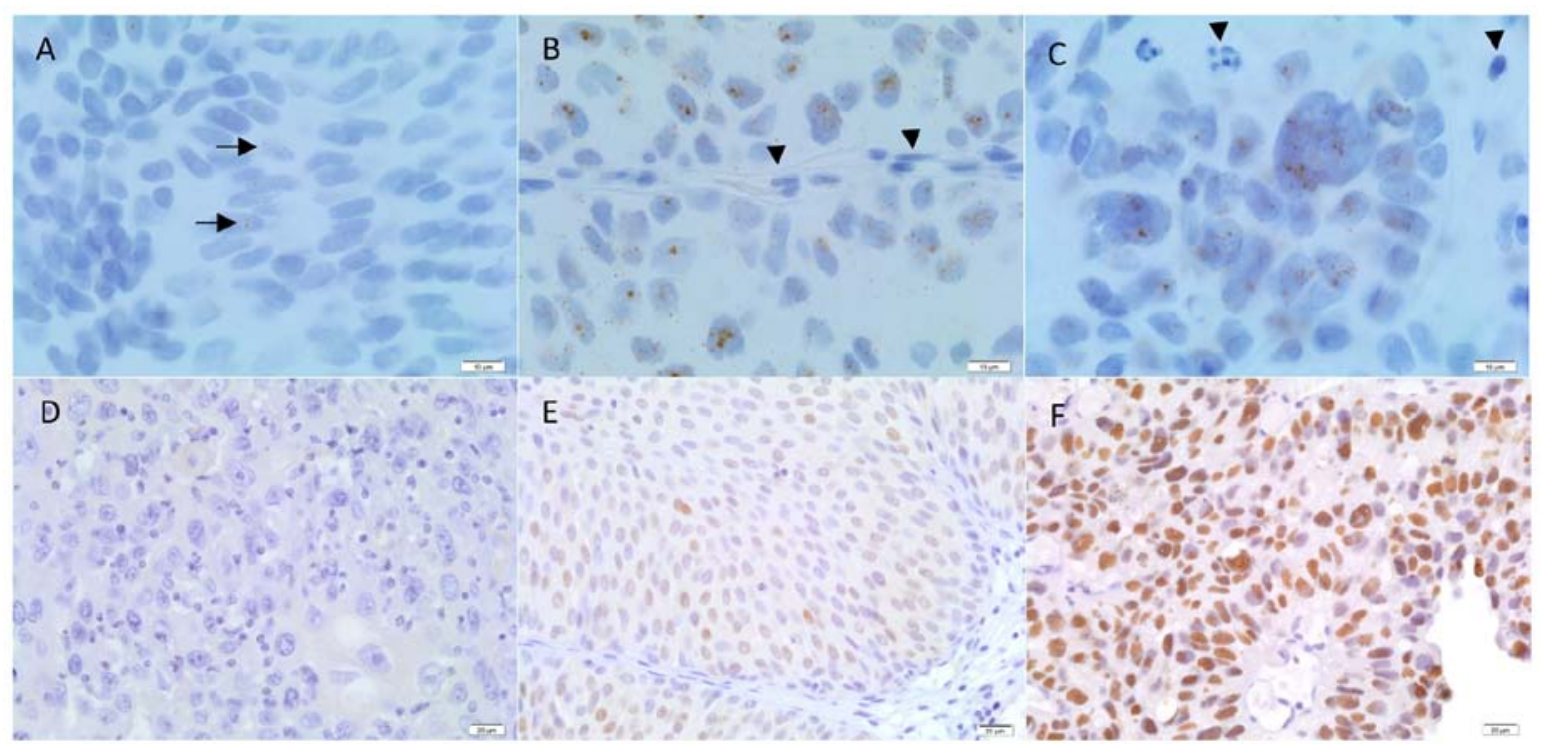

Figure 1. UCA1 RNA chromogenic in situ hybridization and p53 IHC staining in UBC samples. (A-C) UCA1 expression is located in the cytoplasm and the nucleus of urothelial tumor cells: (A) Few dots were observed in few tumor cells (score 1; arrows) and $>10$ dots were homogeneously observed in tumor cells (score 2) of (B) low-grade UBC and (C) high-grade UBC cases. (B and C) No UCAl dot staining was observed in endothelial, stromal or inflammatory cells (arrows). For each UBC case, the mean score of the 6 tissue cores was calculated and the case was categorized as 'UCAl-negative or with low expression' and as 'UCA1-positive or overexpressed' if the mean score was $<1$ and $\geq 1$, respectively. (D-F) Assessment of p53 IHC expression using a three-tier score: (D) 0 (no staining), (E) 1 ( $\leq 25 \%$ of cells with weak, heterogeneous cytoplasmic and nuclear staining) and (F) 2 ( $>25 \%$ of cells with high homogeneous nuclear staining). UCA1, urothelial carcinoma-associated 1; UBC, urothelial bladder cancer; IHC, immunohistochemistry.
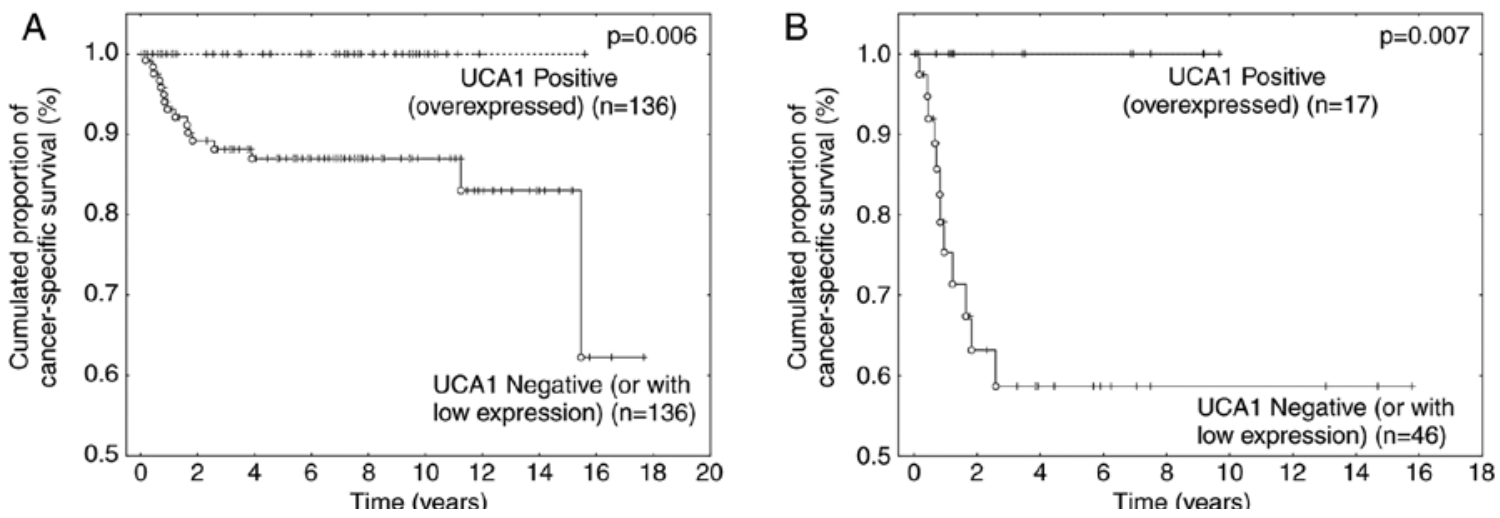

Figure 2. Prognostic value of UCAl expression in UBC. Kaplan-Meier plots indicating cancer-specific survival of patients stratified by UCA1 expression categorized as 'UCA1-negative or with low expression' and as 'UCA1-positive or overexpressed' in (A) UBC tumor samples (all cases) and (B) only in muscle-invasive bladder cancer. UCA1, urothelial carcinoma-associated 1; UBC, urothelial bladder cancer.

patient age $(\mathrm{P}=0.0003)$, multifocality $(\mathrm{P}=0.03)$, concomitant CIS ( $\mathrm{P}=0.00005)$, lymphovascular tumor cell invasion $(\mathrm{P}<0.00001)$, UBC tumor high-grade $(\mathrm{P}=0.0003)$ and $\mathrm{UBC}$ tumor high-stage $(\mathrm{P}=0.00002)$ were all significantly associated with reduced DFS rate. With the exception of patient age and multifocality, the same clinical factors were all significantly associated with reduced survival rate $(\mathrm{P}<0.01$ and $\mathrm{P}<0.001$ respectively, Table III). Regarding $U C A 1$ expression, no significant impact was observed on DFS (Table III). Conversely, UCA1 overexpression was significantly associated with increased survival rate in UBC patients $(\mathrm{P}=0.006)$, and this result was independent of tumor stage (Fig. 2A and B). Indeed, patients with MIBC that overexpressed $U C A 1$ were associated with a 5-year survival rate of $100 \%$, while the 5-year survival rate was only $58 \%$ for patients whose MIBC failed to overexpress the biomarker ( $\mathrm{P}=0.007$; Fig. $2 \mathrm{~B})$. All patients with $\mathrm{UBC}$ that overexpressed $U C A 1$ in the present series remained alive at the end of this study (Fig. 2A and B). Considering only the UCAl group with 'negative or with low expression', multivariate survival analyses combining tumor stage, concomitant CIS and lymphovascular invasion highlighted a high tumor stage ( $\geq$ pT2) as being the sole independent prognostic factor associated with worse survival rate for UBC patients $(\mathrm{P}=0.003$; data not shown).

Finally, the study investigated whether the expression of UCA1, p53 and Ki-67 was interrelated. As noted in Table IV, UCA1 overexpression was more frequently observed in association with weak and heterogeneous expression of p53 (i.e., a 'p53-wildtype immunoprofile'; $\mathrm{P}=0.003$ ) and with a low $\mathrm{Ki}-67$ proliferative index $(<25 \%$; $\mathrm{P}=0.008)$, compared with the absence of $U C A 1$ overexpression. 


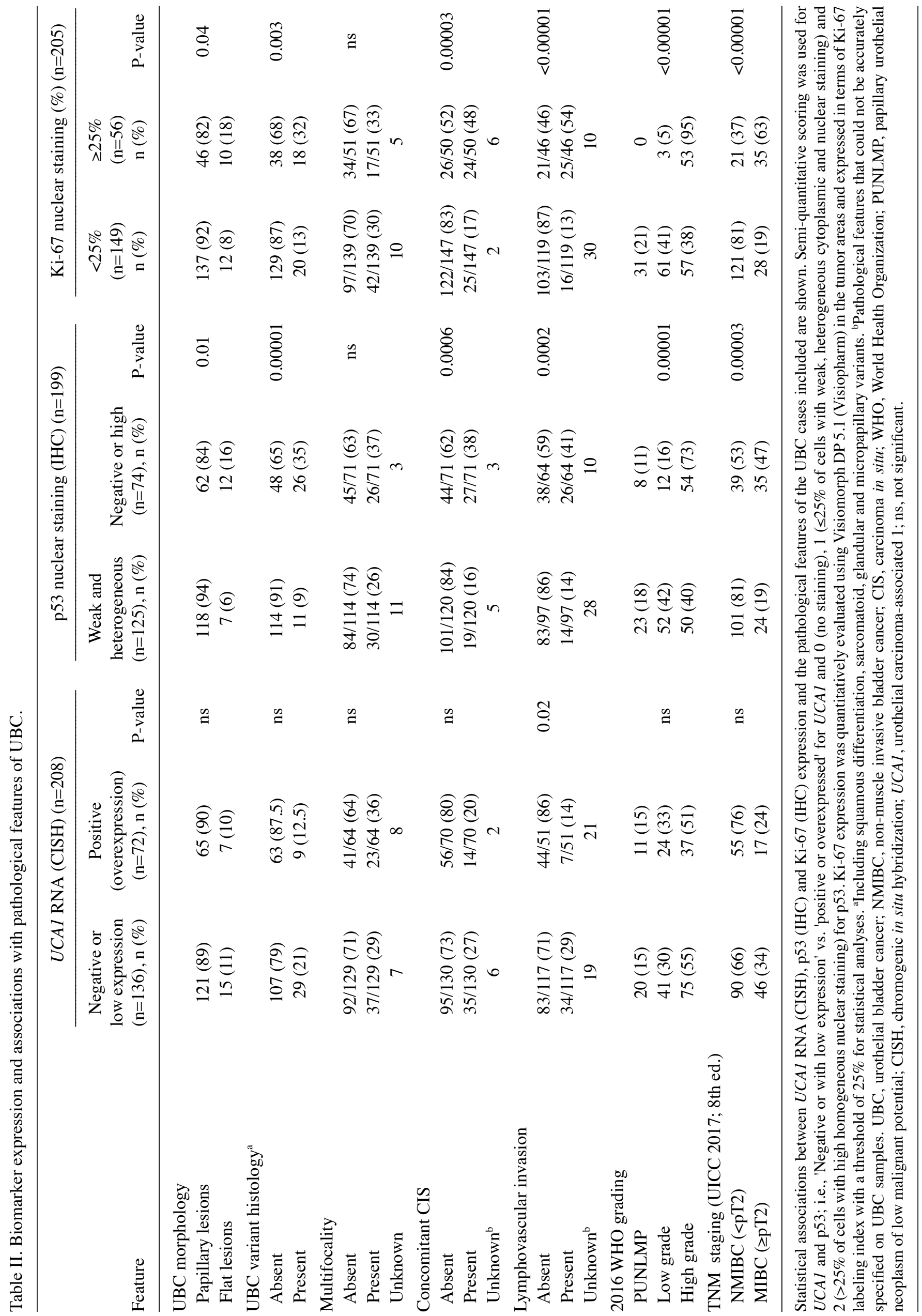


Table III. Univariate survival analyses for patients with UBC.

\begin{tabular}{|c|c|c|}
\hline Feature & $\begin{array}{c}\text { Disease-free } \\
\text { survival, } \\
\text { P-value }\end{array}$ & $\begin{array}{c}\text { Cancer-specific } \\
\text { survival, } \\
\text { P-value }\end{array}$ \\
\hline Age, years $^{\mathrm{a}}$ & 0.0003 & ns \\
\hline \multicolumn{3}{|l|}{ UBC morphology } \\
\hline \multicolumn{3}{|l|}{ Papillary lesions } \\
\hline Flat lesions & ns & ns \\
\hline \multicolumn{3}{|l|}{ UBC variant histology $\mathrm{y}^{\mathrm{b}, \mathrm{c}}$} \\
\hline \multicolumn{3}{|l|}{ Absent } \\
\hline Present & ns & ns \\
\hline \multicolumn{3}{|l|}{ Multifocality ${ }^{\mathrm{b}}$} \\
\hline \multicolumn{3}{|l|}{ Absent } \\
\hline Present & 0.03 & ns \\
\hline \multicolumn{3}{|l|}{ Concomitant CIS ${ }^{\mathrm{b}}$} \\
\hline \multicolumn{3}{|l|}{ Absent } \\
\hline Present & 0.00005 & $<0.00001$ \\
\hline \multicolumn{3}{|l|}{ Lymphovascular invasion $^{\mathrm{b}}$} \\
\hline \multicolumn{3}{|l|}{ Absent } \\
\hline Present & $<0.00001$ & 0.0006 \\
\hline \multicolumn{3}{|l|}{2016 WHO grading ${ }^{b}$} \\
\hline \multicolumn{3}{|l|}{ Low grade } \\
\hline High grade & 0.0003 & 0.002 \\
\hline \multicolumn{3}{|l|}{ TNM staging } \\
\hline \multicolumn{3}{|l|}{ (UICC 2017; 8th ed.) $^{\mathrm{b}}$} \\
\hline \multicolumn{3}{|l|}{ NMIBC (<pT2) } \\
\hline MIBC ( $\geq$ pT2) & 0.00002 & $<0.00001$ \\
\hline \multicolumn{3}{|l|}{$U C A 1$ overexpression $^{\mathrm{b}}$} \\
\hline \multicolumn{3}{|l|}{ Negative or low expression } \\
\hline Positive (overexpression) & ns & 0.006 \\
\hline
\end{tabular}

The table describes the univariate statistical survival analyses of well-known prognostic features for patients with UBC in our series. Each variable is considered as either a continuous variable in ${ }^{a}$ univariate Cox regression or a ${ }^{b}$ two-class factor analyzed by the log-rank test. 'Including squamous differentiation, sarcomatoid, glandular and micropapillary variants. UBC, urothelial bladder cancer; NMIBC, non-muscle invasive bladder cancer; CIS, carcinoma in situ; WHO, World Health Organization; ns, not significant.

\section{Discussion}

Advances in the sequencing of the human genome led to the determination that protein-coding genes compose $<3 \%$ of the human genome. Yet $>80 \%$ of genes are actively transcribed to RNA without protein-coding potential, referred to as ncRNAs (5). Accumulating evidence has shown that lncRNAs are often altered in urological cancer types, notably in the prostate and kidneys, and in UBC $(25,26)$. Among these lncRNAs, $U C A 1$ was originally reported to be involved in UBC carcinogenesis, promoting in vitro tumorigenicity and invasive behavior (10-12) Moreover, recent meta-analyses investigating the association between the expression levels of UCAl and prognosis (using RT-qPCR methodology approaches) noted that $U C A 1$ was implicated in the biology of other solid tumors, including gastric, colorectal, lung, breast and ovarian carcinoma $(13,14)$. Data from those meta-analyses (mainly conducted in Chinese patients) concluded a global positive association between a high expression level of UCAl RNA and tumor stage, lymph node metastasis and poor survival $(13,14)$. Taking into account the previous in vitro results regarding UCA1 involvement in the carcinogenesis of UBC cell lines, it was notable and unexpected that these meta-analyses did not included studies on patients with UBC $(13,14)$. In 2017 , Droop et al (15) reported the RT-qPCR expression levels of several lncRNAs (including $U C A 1$ ) in a series of $106 \mathrm{UBC}$ cases in order to assess the correlation with clinicopathological parameters, including tumor grade, tumor stage and patient survival; the data of the publicly available bladder urothelial carcinoma dataset from The Cancer Genome Atlas (TCGA) was also analyzed (15). In the series, it was found that patients with high $U C A 1$ expression experienced considerably better overall survival rate compared with that of patients with low levels of $U C A l$ expression, but this result was not confirmed in the TCGA dataset (15). Notably, the present study confirmed this result in a larger series of UBC cases and using another methodology (CISH instead of RT-qPCR). Indeed, it was shown that patients whose UBC overexpressed UCAl were associated with improved overall survival rate compared with that of patients whose UBC failed to overexpress this biomarker, and this result was significantly maintained in the aggressive group of MIBC. In the present series, no patient with MIBC that overexpressed $U C A 1$ succumbed during the follow-up ( $>5$ years), as opposed to a 5-year survival rate of $58 \%$ in the group with UBC that did not overexpress $U C A 1$. Notably [and similarly to Droop et al (15)], no statistical association was found between UCA1 overexpression and known pathological prognostic factors in UBC, including tumor architecture (papillary vs. flat UBC), presence of a histological secondary variant, tumor multifocality, concomitant CIS, tumor grade and tumor stage. By consequence, UCAl overexpression appears to be a potential novel independent molecular biomarker associated with an improved CSS in patients with UBC in general, and with MIBC, in particular. This result opposes data provided by Wang et al (7) in 2006, which positively associated the expression of UCA1 in $46 \mathrm{UBC}$ cases with tumor stage, grade and multicentricity. However, precise data regarding UBC sample characterization and patient follow-up were not available in the study manuscript or supplementary data. The present results were also strengthened by the negative association between $U C A 1$ expression and two biomarkers (Ki-67 and p53) associated with UBC aggressiveness. Indeed, UBC that overexpressed UCA1 more often presented a low Ki-67 proliferative index and a p53 'wild-type' immunoprofile and thus behaved less aggressively as compared with UBC that failed to overexpress $U C A 1$.

In the present study, the CISH methodology was selected instead of RT-qPCR as CISH enables the gathering of genetic information in the context of tissue morphology, i.e., the tumor cells and their microenvironment. This methodology is currently used in pathology labs to improve patient management (27). UCA1 signal dots was detected in $80 \%$ of all the UBC samples, but clear UCAI overexpression was only 


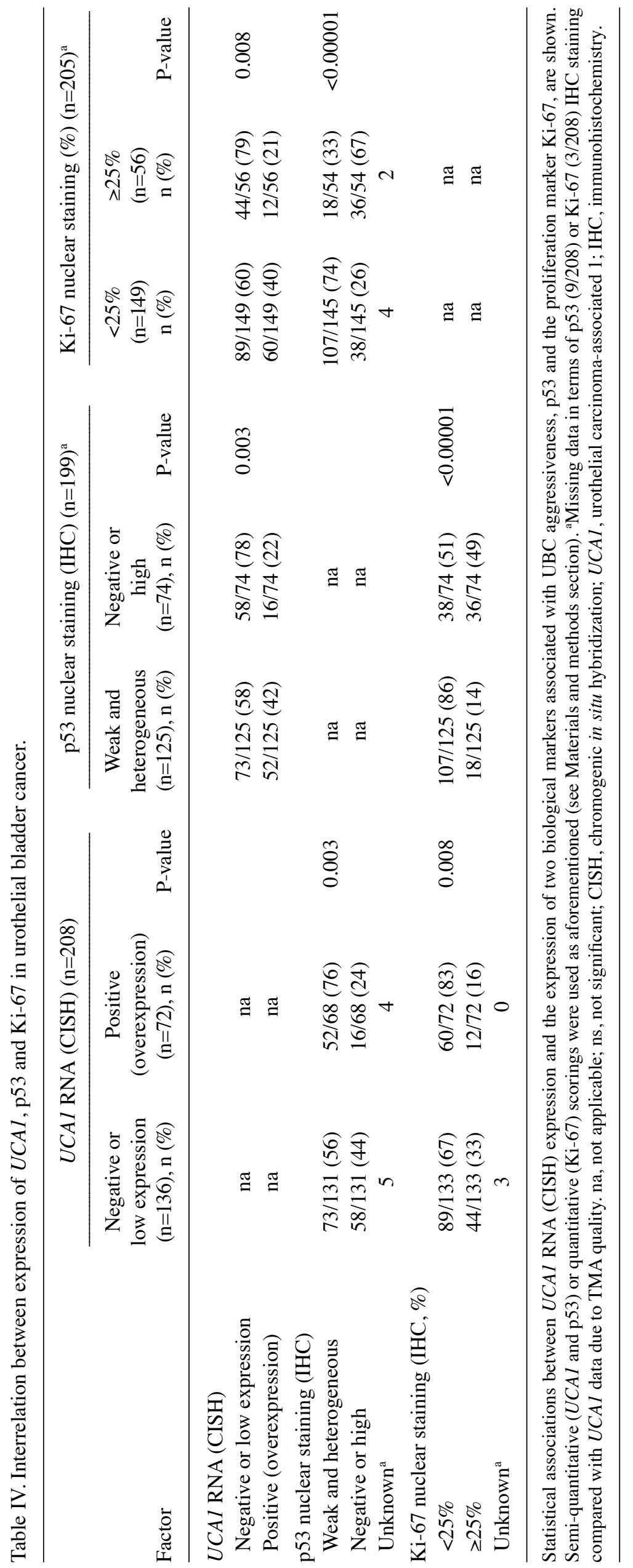


observed in $35 \%$ of them. As illustrated in Fig. 1B-C, UCAI overexpression was only considered when numerous $U C A I$ dots were observed in urothelial tumor cells, and these results were concordantly obtained by two different pathologists. Consequently, the positivity threshold for CISH used in the present study should be easily applied by pathologists in daily practice for the management of patients with UBC.

Genes and pathways that are key drivers of UBC have been identified by previous genome-wide expression and sequencing studies, and a complex landscape with numerous molecular subclasses that travel across conventional tumor grade and stage have been revealed $(3,28)$. UBC are genomically heterogeneous, with frequent alterations in genes regulating receptor kinase signaling, cell cycle control and chromatin state, resulting in distinct clinical outcomes (29). Using next-generation sequencing, Kim et al (29) identified $P I 3 K / A K T$ pathway alterations in $35 \%$ of UBC cases, and noted that in UBC patients treated with radical cystectomy, $P I K 3 C A$ mutation or $P I 3 K / A K T$ pathway alterations are associated with a significant favorable prognosis, whereas TP53 and CDKN2A alterations are associated with poor outcomes. Notably, two recent publications linked UCA 1 to PI3K/AKT pathway activation (12), but also to $C D K N 2 A-p 16 I N K$ mRNA stabilization (30). Yang et al (12) showed that $U C A 1$ stimulated cell cycle progression by increasing CREB expression via activation of the PI3K/AKT pathway in vitro in the human bladder cancer BLZ-211 cell line, with UCAl expression being positively correlated with AKT1 expression and AKT phosphorylation. In 2014, Kumar et al (30) demonstrated that UCA1 may act as a tumor suppressor gene, as its overexpression of $U C A 1$ was able to induce cellular senescence at least partially by the disruption of pl6INK mRNA and hnRNPA1 interactions, resulting in increased CDKN2A-pl6INK mRNA stability (30). In accord with this study, the present study showed that the overexpression of UCAl was more frequently observed in association with a low Ki-67 proliferative index. Taken together, these previous studies appear to be in line with the present results suggesting that $U C A 1$ overexpression should be associated with less aggressive bladder tumors.

In conclusion, the present study provides novel evidence regarding $U C A 1$ expression in urothelial tumor cells and its involvement in UBC carcinogenesis. The results highlight the independent contribution of $U C A I$ overexpression towards improved outcomes for patients with UBC. The findings confirm the large heterogeneity that composes the MIBC group and may open novel avenues in order to better stratify patients with regards to management and treatment.

\section{Acknowledgements}

The authors would like to thank their collaborators from the Erasme Hospital Biobank (Brussels, Belgium; BE_BERA1; Biobanque Hôpital Erasme-ULB (BERA); BE_NBWB1; Biothèque Wallonie Bruxelles (BWB); BBMRI-ERIC) for having made available a large number of UBC samples. The authors would particularly like to thank DiaPath, part of the Center for Microscopy and Molecular Imaging (CMMI, Charleroi, Hainaut, Belgium) for TMA manufacturing, CISH and IHC experiments, and biomarker quantification.

\section{Funding}

Funding for the present study was from Fonds Yvonne Boël (Brussels, Belgium; http://www.fondsyvonneboel.be/). The Center for Microscopy and Molecular Imaging is supported by the European Regional Development Fund (http://ec.europa. eu/regional_policy/thefunds/regional/index_en.cfm) and the Walloon Region (http://www.wallonie.be/). The funders had no role in study design, data collection and analysis, decision to publish or preparation of the manuscript.

\section{Availability of data and materials}

All data generated or analyzed during this study are included in this published article.

\section{Authors' contributions}

LL and DM: Constitution of the clinical series, validation of quality controls and biomarkers quantification, and assistance in writing the manuscript. MLM: Implementation of the methodology for setting up UCAl detection by CISH and assiatnce in writing the manuscript. JA: TMA manufacturing, IHC and CISH experiments, quality control validation, and assistance in writing the manuscript. YRVE: Slide digitalization and computer-assisted quantitative analyses of KI-67 labeling index using Visiomorph DP 5.1. TR: Assistance with clinical case selection. CD: Statistical analyses and writing of the manuscript. IS: Data analyses and assistance in writing the manuscript. SR: Case reviews and classifications, biomarker quantification, data analyses and manuscript writing.

\section{Ethics approval and consent to participate}

The present study was approved by the Ethical Committee of the Erasme University Hospital (Brussels, Belgium; ref., P2015/041).

\section{Patient consent for publication}

According to Belgian law, no written informed consent was required for archival material in the context of retrospective studies. The ethical committee thus waived the requirement for written informed consent from the participant.

\section{Competing interests}

The authors declare that they have no competing interests.

\section{References}

1. Babjuk M,Böhle A, Burger M,Capoun O,Cohen D,CompératEM, Hernández V, Kaasinen E, Palou J, Rouprêt M, et al: EAU guidelines on non-muscle-invasive urothelial carcinoma of the bladder: Update 2016. Eur Urol 71: 447-461, 2017.

2. Alfred Witjes J, Lebret T, Compérat EM, Cowan NC, De Santis M, Bruins HM, Hernández V, Espinós EL, Dunn J, Rouanne M, et al: Updated 2016 EAU guidelines on muscle-invasive and metastatic bladder cancer. Eur Urol 71: 462-475, 2017.

3. Knowles MA and Hurst CD: Molecular biology of bladder cancer: New insights into pathogenesis and clinical diversity. Nat Rev Cancer 15: 25-41, 2015. 
4. Sjödahl G, Lauss M, Lövgren K, Chebil G, Gudjonsson S, Veerla S, Patschan O, Aine M, Fernö M, Ringnér M, et al: A molecular taxonomy for urothelial carcinoma. Clin Cancer Res 18: 3377-3386, 2012.

5. Yang G, Lu X and Yuan L: LncRNA: A link between RNA and cancer. Biochim Biophys Acta 1839: 1097-1109, 2014.

6. Zhang Q, Su M, Lu G and Wang J: The complexity of bladder cancer: Long noncoding RNAs are on the stage. Mol Cancer 12: $101,2013$.

7. Wang XS, Zhang Z, Wang HC, Cai JL, Xu QW, Li MQ, Chen YC, Qian XP, Lu TJ, Yu LZ, et al: Rapid identification of UCA1 as a very sensitive and specific unique marker for human bladder carcinoma. Clin Cancer Res 12: 4851-4858, 2006.

8. Milowich D, Le Mercier M, De Neve N, Sandras F, Roumeguere T, Decaestecker C, Salmon I and Rorive S: Diagnostic value of the UCA1 test for bladder cancer detection: A clinical study. Springerplus 4: 349, 2015.

9. Srivastava AK, Singh PK, Rath SK, Dalela D, Goel MM and Bhatt ML: Appraisal of diagnostic ability of UCA1 as a biomarker of carcinoma of the urinary bladder. Tumour Biol 35 11435-11442, 2014.

10. Xue M, Li X, Li Z and Chen W: Urothelial carcinoma associated 1 is a hypoxia-inducible factor-1 $\alpha$-targeted long noncoding RNA that enhances hypoxic bladder cancer cell proliferation, migration, and invasion. Tumour Biol 35: 6901-6912, 2014.

11. Wang X, Gong Y, Jin B, Wu C, Yang J, Wang L, Zhang Z and Mao Z: Long non-coding RNA urothelial carcinoma associated 1 induces cell replication by inhibiting BRG1 in 5637 cells. Oncol Rep 32: 1281-1290, 2014.

12. Yang $\mathrm{C}$, Li X, Wang Y, Zhao L and Chen W: Long non-coding RNA UCA1 regulated cell cycle distribution via CREB through PI3-K dependent pathway in bladder carcinoma cells. Gene 496 8-16, 2012.

13. Liu FT, Zhu PQ, Luo HL, Zhang Y and Qiu C: Prognostic value of long non-coding RNA UCA1 in human solid tumors. Oncotarget 7: 57991-58000, 2016

14. Wang X, Peng F, Cheng L, Yang G, Zhang D, Liu J, Chen X and Zhao S: Prognostic and clinicopathological role of long non-coding RNA UCA1 in various carcinomas. Oncotarget 8: 28373-28384, 2017.

15. Droop J, Szarvas T, Schulz WA, Niedworok C, Niegisch G, Scheckenbach K and Hoffmann MJ: Diagnostic and prognostic value of long noncoding RNAs as biomarkers in urothelial carcinoma. PLoS One 12: e0176287, 2017.

16. Ding W, Gou Y, Sun C, Xia G, Wang H, Chen Z, Tan J, Xu K and Qiang D: Ki-67 is an independent indicator in non-muscle invasive bladder cancer (NMIBC); combination of EORTC risk scores and $\mathrm{Ki}-67$ expression could improve the risk stratification of NMIBC. Urol Oncol 32: 42.e13-e19, 2014.

17. Margulis V, Shariat SF, Ashfaq R, Sagalowsky AI and Lotan Y: $\mathrm{Ki}-67$ is an independent predictor of bladder cancer outcome in patients treated with radical cystectomy for organ-confined disease. Clin Cancer Res 12: 7369-7373, 2006.
18. Moch H, Humphrey PA, Ulbright TM and Reuter VE: WHO Classification of Tumours of the Urinary System and Male Genital Organs. 4th edition. IARC, Lyon 8, 2016.

19. Brierley JD, Gospodarowicz MK and Wittekind C (eds) The TNM Classification of Malignant Tumours, 8th edition. Union for International Cancer Control (UICC); Wiley-Blackwell, 2017.

20. Decaestecker C, Lopez XM, D'Haene N, Roland I, Guendouz S, Duponchelle C, Berton A, Debeir O and Salmon I: Requirements for the valid quantification of immunostains on tissue microarray materials using image analysis. Proteomics 9: 4478-4494, 2009.

21. Rorive S, Lopez XM, Maris C, Trepant AL, Sauvage S, Sadeghi N, Roland I, Decaestecker C and Salmon I: TIMP-4 and CD63: New prognostic biomarkers in human astrocytomas. Mod Pathol 23: 1418-1428, 2010

22. Talhouk A, McConechy MK, Leung S, Li-Chang HH, Kwon JS, Melnyk N, Yang W, Senz J, Boyd N, Karnezis AN, et al: A clinically applicable molecular-based classification for endometrial cancers. Br J Cancer 113: 299-310, 2015.

23. Kurman RJ, Carcangiu ML, Herrington CS and Young RH: WHO classification of tumors of female reproductive organs. 4th edition. IARC, Lyon 6, 2014.

24. Mota A, Triviño JC, Rojo-Sebastian A, Martínez-Ramírez A Chiva L, González-Martín A, Garcia JF, Garcia-Sanz P and Moreno-Bueno G: Intra-tumor heterogeneity in TP53 null high grade serous ovarian carcinoma progression. BMC Cancer 15: 940, 2015.

25. Martens-Uzunova ES, Böttcher R, Croce CM, Jenster G, Visakorpi $\mathrm{T}$ and Calin GA: Long noncoding RNA in prostate, bladder, and kidney cancer. Eur Urol 65: 1140-1151, 2014.

26. Xue M, Chen W and Li X: Urothelial cancer associated 1: A long noncoding RNA with a crucial role in cancer. J Cancer Res Clin Oncol 142: 1407-1419, 2016.

27. Guhley ML and Tang W: Laboratory assays for Epstein-Barr virus-related disease. J Mol Diagn 10: 279-292, 2008.

28. Aine M, Eriksson P, Liedberg F, Höglund M and Sjödahl G: On molecular classification of bladder cancer: Out of one, many. Eur Urol 68: 921-923, 2015.

29. Kim PH, Cha EK, Sfakianos JP, Iyer G, Zabor EC, Scott SN, Ostrovnaya I, Ramirez R, Sun A, Shah R, et al: Genomic predictors of survival in patients with high-grade urothelial carcinoma of the bladder. Eur Urol 67: 198-201, 2015.

30. Kumar PP, Emechebe U, Smith R, Franklin S, Moore B, Yandell M, Lessnick SL and Moon AM: Coordinated control of senescence by lncRNA and a novel T-box 3 co-repressor complex. Elife 3, 2014. International (CC BY-NC-ND 4.0) License. 